\title{
Daily Living Performance in Schizophrenic Patients in Correlation with Positive and Negative Symptoms
}

\author{
Nurul Amelia Rahayu Putri, ${ }^{1}$ Tuti Kurnianingsih, ${ }^{2}$ Santi Andayani ${ }^{2}$ \\ ${ }^{1}$ Faculty of Medicine Universitas Padjadjaran, Indonesia, ${ }^{2}$ Department of Psychiatry Faculty of \\ Medicine Universitas Padjadjaran/Dr Hasan Sadikin General Hospital Bandung, Indonesia
}

\section{Abstract}

Background: Schizophrenia is a global mental health problem. Patients experience problems in their daily-living performance. Positive and negative symptoms have been part of characteristics of schizophrenia that are absent in normal individuals, which include a distinctive fundamental distortion of thoughts and perception (positive symptoms) and loss of normal function and behavior (negative symptoms). This study aimed to explore the correlation between positive and negative symptoms and daily-living performance in schizophrenia.

Methods: A correlative analytical study with a cross-sectional approach was conducted using secondary data from the Outpatient Clinic of the Mental Hospital of West Java Province. Data on socio-demographic characteristics were collected and positive and negative symptoms as assessed by Positive and Negative Syndrome Scale or Subscales (PANSS) were correlated with the activities of daily living (ADLs) and instrumental activities of daily living (IADLs), which were further analyzed by Spearman's correlation test.

Results: In total, 31 male schizophrenic patients aged between 24-45 years old. There was no significant correlation between ADLs dependency levels and both positive and negative symptoms. However, there was a significant correlation between IADLs function and positive symptoms and not with negative symptoms.

Conclusions: Even though no correlation was identified between ADLs dependency levels with positive and negative symptoms, the ADLs and IADLs assessments should be considered as important tools for schizophrenic patients for rehabilitation to improve daily-living functioning.

Keywords: Barthel index, lawton-brody scale, negative syndrome scale, positive syndrome scale

\section{Introduction}

Schizophrenia is a global mental health problem with high costs and needs for care. ${ }^{1}$ According to national health basic research (Riset Kesehatan Dasar, Riskesdas) year 2013, the prevalence of psychotic disorders in Indonesia has reached 1.7 per mile area. West Java is ranked $11^{\text {th }}$ among the provinces in Indonesia with prevalence as high as 1.6 per mile area. ${ }^{2}$ The prevalence of schizophrenia has its peak at around 40 years of age, with a decline in the older age group, whereas the incidence is higher among males.,4 Disability adjusted life year (DALY) has been used to measure disease burden, based on the definition of Global Burden of Disease. ${ }^{3}$ The DALY is calculated by summing years of life lived with disability (YLDs) and years of life lost (YLLs) to premature mortality for a disease. Schizophrenia is being the most disabling disorder in $12^{\text {th }}$ rank among 310 diseases and injuries globally. ${ }^{3}$

Positive symptoms are a distinctive fundamental distortion of thoughts and perception, including hallucinations and delusions. Negative symptoms are deficits or loss of normal function and behavior, such as anhedonia, avolition, asocial,bluntaffect, alogia. Both symptoms are distinctive characteristics of schizophrenia that are absent in normal individuals. Along with it, schizophrenic patients experience disturbances on activities of daily living (ADLs) and instrumental activities of daily living (IADLs)..$^{5,6}$ Activities of daily living (ADLs) are activities to fulfill

Correspondence: Nurul Amelia Rahayu Putri, Faculty of Medicine, Universitas Padjadjaran, Jalan Raya BandungSumedang Km.21, Jatinangor, Sumedang, Indonesia, E-mail: nurul16006@mail.unpad.ac.id 
physical needs, including grooming, clothing, urinating/defecating, walking, and feeding. ${ }^{7}$ Instrumental activities of daily living comprise of more complex activities for independent living, such as managing finances and medication. ${ }^{7}$ Moreover, the absence of similar studies in Indonesia, and inconsistent results from previous studies require further research to correlate the positive and negative symptoms with daily-living performance, specifically ADLs and IADLs. ${ }^{8-10}$ Therefore, this study aimed to explore the correlation between positive and negative symptoms and daily-living performance in schizophrenia.

\section{Methods}

This study was a correlative analytical study with a cross-sectional approach, conducted at the Outpatient Clinic of Psychiatry in Mental Hospital of West Java Province. This study used secondary data from the previous study "Correlation between Vitamin D Serum in Schizophrenic Patients and Positive and Negative Symptoms Subscale". This study had ethical clearance exemption from the Research Ethics Committee, Universitas Padjadjaran No. 1211/UN6.KEP/EC/2019.

In brief, data were collected from patients who came for control treatment from December 2018 to February 2019. In order to calculate the sample size, the formula for finding a correlation between positive and negative symptoms and ADLs, as well as IADLs, was employed using error percentage of $5 \%$, test strength of $90 \%$, an expected minimum correlation coefficient of $0.55 .{ }^{8}$ The estimated sample size was 31 samples. Patients were included based on the gender which was male. The patients need to be calm and cooperative, justified by PANSS-EC Score $<25$. The exclusion criteria were patients with physical disabilities, including neuromuscular diseases, paralysis, deformity on limbs, disfigurements, blindness and deafness, and incomplete data.

Data on positive and negative symptoms of psychopathology were collected. The positive and negative symptoms of psychopathology were assessed using the Positive and Negative Syndrome Scale or Subscales (PANSS), administered by trained raters, as published earlier. The scale had good and strong psychometric properties in terms of reliability, validity, and sensitivity. ${ }^{11}$ The single items of the PANSS were rated on a 7 -point scale $(1=$ absent, $2=$ minimal, $3=$ mild, $4=$ moderate, $5=$ moderatesevere, $6=$ severe, and $7=$ extreme). ${ }^{12}$ The scoring of PANSS was performed by adding every item of each subscale with a potential range of 7-49.

The daily living performance was measured using the Barthel ADL Index and the LawtonBrody IADL Scale. Barthel ADL Index assessed the mobility function, continence, and selfcare such as eating, bathing, and dressing, whereas the Lawton-Brody IADL Scale assessed the ability to use tools or interaction with other people or the environment, such as preparing the meal, using the telephone, shopping, and housekeeping. The potential range for both the Barthel ADL Index and the Lawton-Brody IADL Scale for males was 0 to 20 and 0 to 5, respectively. Based on Barthel ADL Index, activities of daily living (ADLs) performance was classified into five levels of ADLs dependency, comprised of independency (20); mild dependency (12-19); moderate dependency (9-11); severe dependency (58); and total dependency (0-4). Lawton-Brody IADL Scale classifies IADLs performance for men into 2 categories: high function or independency (5), and low function or dependency $(0-4) \cdot{ }^{12}$

Statistical analysis was executed using a computed statistical program (SPSS v. 22, licensed to Universitas Padjadjaran). Sociodemographic characteristics were analyzed using descriptive statistics. Correlation between positive and negative symptoms and ADLs were analysed with Spearman's correlation test and $\mathrm{p}<0.05$ considered as statistically significant. Correlation between positive and negative symptoms and IADLs were analyzed with Spearman's correlation test and $\mathrm{p}<0.05$ considered as statistically significant, after analysis of the distribution of data with the Shapiro-Wilk test.

\section{Results}

In total, data of 31 male schizophrenic patients aged between 24 to 45 years old were collected with a mean age of 35.2 years old (s.d. 6.7 years). Most of them were unmarried (22/31), employed (17/31), and had high school education (20/31) at the time of the study. These patients had never been admitted to the hospital (11/31) and almost half of them were admitted more than once $(13 / 31)$. More than half of the patients experienced chronicity of the illness (19/31). Moreover, patients demonstrated independency $(21 / 31)$ and the rest revealed mild dependency $(10 / 31)$. The patients showed high IADLs function $(21 / 31)$, and only some were low (10/31) as shown in Table 1. 
Table 1 Socio-demographic and Clinical Characteristics of Male Schizophrenic Patients $(n=31)$ at the Outpatient Clinic of Psychiatry in Mental Hospital of West Java Province, Recruited from December 2018-February 2019

\begin{tabular}{lc}
\hline \multicolumn{1}{c}{ Characteristics } & $\mathrm{n}(\%)$ \\
\hline Marital status & $8(26)$ \\
Married & $22(71)$ \\
Unmarried & $1(3)$ \\
Divorced & \\
Occupational Status & $17(55)$ \\
Employed & $14(45)$ \\
Unemployed & \\
Education level & $2(6)$ \\
No education & $4(13)$ \\
Primary school & $3(10)$ \\
Secondary/middle school & $20(65)$ \\
High school & $2(6)$ \\
College/university & \\
History of hospitalization & $11(35)$ \\
Never & $7(23)$ \\
Once (1 admission) & $13(42)$ \\
More than once (> 1 admission) & \\
Duration of illness & $12(39)$ \\
2-5 years & $19(61)$ \\
$>$ 5 years & $10(32)$ \\
BAI & $21(68)$ \\
Mild dependency & $10(32)$ \\
Independency & $21(68)$ \\
LIADL & \\
Low function (dependency) & \\
\hline High function (independency) & \\
\hline BAI-Bathel & \\
\hline
\end{tabular}

Note: BAI=Barthel activity of daily living index; LIADL=Lawton-Brody instrumental activities of daily living scalet

The PANSS-Positive Subscale subdomains score in the samples revealed a median of 3 , except for subdomain excitement and grandiosity, both had 1 as the median. The PANSS-Negative Subscale subdomains score in the samples also revealed the median of 3 , with the exception of subdomain stereotyped thinking, with 2 as its median (Table 2).

Before statistical correlation analyses, the Shapiro-Wilk test was used to test the normality of numeric data distribution. Both PANSS-Positive Subscale and PANSS-Negative Subscale had normal data distribution ( $p$-value
$=0.350$, and $p$-value $=0.759$, respectively); whereas, LIADL did not demonstrate normal data even with data transformation ( $p$-value $=0.00$ ).

Linearity test was conducted between Barthel ADL Index (BAI) and PANSSPositive Subscale, and PANSS-Negative Subscale, resulting in the linear relationship ( $p$-value $=0.425$, and $p$-value $=0.809$, respectively). Linearity test was also operated between LIADL and PANSS-Positive Subscale, and PANSS-Negative Subscale, and showed a linear relationship ( $\mathrm{p}$-value $=0.512$, and $\mathrm{p}$-value $=$ 
Table 2 PANSS-Positive Subscale and PANSS-Negative Subscale of Male Schizophrenic Patients $(n=31)$ at the Outpatient Clinic of Psychiatry in Mental Hospital of West Java Province Recruited during December 2018-February 2019.

\begin{tabular}{lc}
\hline \multicolumn{1}{c}{ Subdomains } & Median (min-max) \\
\hline PANSS-positive subscale & $3(1-6)$ \\
Delusions & $3(1-6)$ \\
Conceptual disorganization & $3(1-5)$ \\
Hallucinatory behavior & $1(1-2)$ \\
Excitement & $1(1-5)$ \\
Grandiosity & $3(1-5)$ \\
Suspiciousness & $3(1-4)$ \\
Hostility & $16.7(6.1)$ \\
Subscale total, M (SD) & \\
PANSS-negative subscale, median (min-max) & $3(2-6)$ \\
Blunted affect & $3(1-5)$ \\
Emotional withdrawal & $3(1-5)$ \\
Poor rapport & $3(1-5)$ \\
Passive-apathetic social withdrawal & $3(1-7)$ \\
Difficulty in abstract thinking & $3(1-6)$ \\
Lack of spontaneity and flow of conversation & $2(1-5)$ \\
Stereotyped thinking & $20.8(5.9)$ \\
Subscale total, M (SD) & Note: PANSS=positive and negative syndrome scale; Min=minimum; Max=maximum; M=mean; SD=standard deviation
\end{tabular}

\subsection{6, respectively).}

A Spearman's correlation was run to determine the relationship between BAI and PANSS-Positive Subscale. There was no correlation between ADLs dependency levels and positive symptoms ( $\mathrm{rs}=0.039 ; \mathrm{p}>0.05$ ); furthermore, there was no correlation between ADLs dependency levels and negative symptoms ( $r s=0.228 ; p>0.05$ ) (Table 3 ).

A Spearman's correlation was run to determine the relationship between LIADL and PANSS-Positive Subscale. There was a correlation between IADLs function and positive symptoms $(\mathrm{rs}=-0.386$; $\mathrm{p}<0.05)$; and there was no correlation between LIADL and negative symptoms ( $\mathrm{rs}=-0.169 ; \mathrm{p}>0.05$ ). (Table 4).

\section{Discussion}

This study has correlated positive and negative symptoms with daily-living performance in schizophrenia. Most of the patients are unmarried and employed, similar to a study in India, ${ }^{9}$ but different from a study in Israel ${ }^{8}$, of whom the majority was single (74\%) and unemployed $(65.4 \%)$, even though the patients have had high school education of 4 to 17 years of education $(\mathrm{M}=11.41, \mathrm{SD}=2.48) .{ }^{8}$ On the contrary, the study in India ${ }^{9}$ has a lower education level, which is a secondary school, suggesting that unemployment has no correlation with the education but rather to the severity of the disease itself.

More than one-fourth of the patients in our study have never been admitted to the hospital (11/31), while almost the same percentage has been admitted more than once $(13 / 31)$. Most of the patients are hospitalized about 5 times. ${ }^{14}$ More than half of the patients experienced chronicity of illness (19/31), similar to a study in Australia. ${ }^{13}$ Most of them show independency, ${ }^{10}$ with high IADLs function, although another study shows conflicting result. ${ }^{9}$

Our study has revealed that the PANSSpositive subscale score (mean $16.7, \mathrm{SD}=6.1$ ) as well as PANSS-negative subscale score (mean=20.8, SD=5.9) are not similar with the previous study conducted in India. ${ }^{9}$ The 
Table 3 Correlation between Barthel ADL Index with PANSS-Positive and Negative Subscale of Male Schizophrenic Patients (n=31)

\begin{tabular}{lcc}
\hline & \multicolumn{2}{c}{$\begin{array}{c}\text { Bivariate Statistics } \\
\text { (Spearman's Correlation Test) }\end{array}$} \\
\cline { 2 - 3 } & rs & P-value \\
\hline PANSS-positive subscale & 0.039 & 0.836 \\
PANSS-negative subscale & 0.228 & 0.217 \\
\hline
\end{tabular}

Note: PANSS - Positive and negative syndrome scale, rs = Spearman's correlation coefficient

Table 4 Correlation between Total Lawton-Brody IADL Scale Score with PANSS-Positive and Negative Subscale of Male Schizophrenic Patients $(n=31)$

\begin{tabular}{lcc}
\hline & \multicolumn{2}{c}{ Bivariate Statistics } \\
& rs & P-value \\
\cline { 2 - 3 } & $-0.386^{\wedge}$ & 0.032 \\
\hline PANSS-positive subscale & -0.169 & 0.365 \\
PANSS-negative subscale & &
\end{tabular}

Note: Correlation was significant at the 0.05 level (2-tailed), PANSS=positive and negative syndrome scale; IADL= instrumental activities of daily living scale, rs=spearman's correlation coefficient

PANSS-negative subscale score correspond to between mildly ill to moderately ill when compared with the clinical global impressions scale. ${ }^{11}$

Although most previous studies find a correlation between positive symptoms and ADL functioning, 10,13 our study shows no significant correlation between ADLs dependency level and positive symptoms, suggesting that schizophrenic patients with persistent psychosis may be able to retain independence in ADLs, partly due to the consistent finding that cognitive impairments are also not severely associated with the current presence of psychosis and functional capacity performance. ${ }^{15}$ Interestingly, our study result shows no significant correlation between ADLs dependency level and negative symptoms, in contradictory to most former studies. ${ }^{10,16}$ Low ADLs functioning is correlated with apathy subscales of Brief Psychiatric Rating Scale-Expanded (BPRS-E), including emotional withdrawal and blunted affect. ${ }^{10}$ In a present study, the correlation has been demonstrated between IADLs function and positive symptoms, and again this result is conflicting with most of the previous studies, ${ }^{9,17}$ in which the correlation between total LIADL score and the positive subscales of PANSS do not show statistical significance. Most former studies also revealed a correlation between IADL's function and negative symptoms. . $^{15,18}$ Negative symptoms have predicted functioning in IADL. ${ }^{8}$ It implies that the severity of these symptoms are associated with daily-living performance. ${ }^{8}$ However, the present study did not demonstrate a correlation between IADL's function and negative symptoms. Despite these results, ADLs and IADL's assessments should be considered as important tools to assess proper rehabilitation program for schizophrenic patients to improve their dailyliving functioning.

All contradictory results may be attributed to the diversity of the patients or differences in methods of ADLs as well as IADLs evaluation in the former studies. A previous study in Israel ${ }^{8}$ has measured IADLs with a performancebased evaluation tool the Revised Observed Tasks of Daily Living (OTDL-R), whereas in Sri Lanka ${ }^{10}$ used self-report and caregiver-report evaluation tool Activities of Daily Living Scale (ADLS) to measure ADLs. However, combining both self-reported and performance-based measures of daily-living performance may be the best way to fully represent an individual's disability. ${ }^{7}$

The ability to perform ADLs and IADLs are dependent upon cognitive and motor abilities. Capability to perform adequate muscular work, or physical performance, is the strongest predictor of ADL limitations. ${ }^{16}$ Physical performance factors include poor physical health status, represented by rates of obesity and related metabolic comorbidities; socioeconomic challenges; and antipsychotic 
medications use ${ }^{16}$ Other confounding factors that also influence ADLs ability are age, ${ }^{5}$ age of onset, ${ }^{19}$ histories of hospitalization, and duration of illness. ${ }^{14}$ Instrumental activities of daily living ability worsen with cognitive impairment, as do motor skills, reducing the capability to perform adequate muscular work. ${ }^{16}$ Other confounding factors that influence IADLs function, including age, employment, and marital status, ${ }^{9}$ level of education, ${ }^{8}$ age of onset, $^{19}$ histories of hospitalization, and duration of illness. ${ }^{14}$

Further studies focusing on the impact of daily-living performance should be reattempted by using both self-reported and performance-based evaluation tools and thoroughly control confounding factors, i.e. cognitive abilities that may influence the results.

As a conclusion, there is no significant correlation between ADLs dependency levels and positive symptoms, neither with negative symptoms. However, there is a correlation between IADLs function and positive symptoms but not with negative symptoms. The ADLs and IADLs assessments should be considered as important tools for schizophrenic patients for rehabilitation to improve daily-living functioning.

\section{References}

1. Charlson FJ, Ferrari AJ, Santomauro DF, Diminic S, Stockings E, Scott JG, et al. Global epidemiology and burden of schizophrenia: findings from the Global Burden of Disease study 2016. Schizophr Bull. 2018;44(6):1195-203.

2. Badan Penelitian dan Pengembangan Kesehatan Kementerian Kesehatan Republik Indonesia. Riset Kesehatan Dasar (Riskesdas) 2013. Jakarta: Badan Penelitian dan Pengembangan Kesehatan Kementerian Kesehatan RI; 2013.

3. Vos T, Barber RM, Bell B, Bertozzi-Villa A, Biryukov S, Bolliger I, et al. Global, regional, and national incidence, prevalence, and years lived with disability for 301 acute and chronic diseases and injuries in 188 countries, 1990-2013: a systematic analysis for the Global Burden of Disease Study 2013. Lancet. 2015;386(9995):743800.

4. Maisyarah TA, Nugraha GI, Lidyana L. Nutritional status of schizophrenic patients at Department of Psychiatry in Dr. Hasan Sadikin General Hospital. Althea Medical Journal. 2014;1(1):40-7.
5. Viertiö S, Tuulio-Henriksson A, Perälä J, Saarni SI, Koskinen S, Sihvonen M, et al. Activities of daily living, social functioning and their determinants in persons with psychotic disorder. Eur Psychiatry. 2012;27(6):409-15.

6. Carter JW, Parnas J, Urfer-Parnas A, Watson J, Mednick SA. Intellectual functioning and the long-term course of schizophrenia-spectrum illness. Psychol Med. 2011;41(6):1223-37.

7. Mlinac ME, Feng MC. Assessment of Activities of Daily Living, Self-Care, and Independence. Arch Clin Neuropsychol. 2016;31(6):506-16.

8. Lipskaya L, Jarus T, Kotler M. Influence of cognition and symptoms of schizophrenia on IADL performance. Scand J Occup Ther. 2011;18(3):180-7.

9. Samuel R, Thomas E, Jacob KS. Instrumental Activities of Daily Living Dysfunction among People with Schizophrenia. Indian J Psychol Med. 2018;40(2):134-8.

10. Wijesundara H, Dayabandara M, Ellepola A, Hanwella R. Psychopathology in patients with schizophrenia attending a psychiatry outpatient clinic at a tertiary care hospital. Sri Lanka J Psychiatry. 2011;2(1).

11. Leucht S, Barabássy Á, Laszlovszky I, Szatmári B, Acsai K, Szalai E, et al. Linking PANSS negative symptom scores with the Clinical Global Impressions Scale: understanding negative symptom scores in schizophrenia. Neuropsychopharmacology. 2019;44(9):1589-96.

12. Dolai MC, Chakrabarty F. Functional status of the elderly santal people. Indian J Gerontol. 2013;27(4):610-20.

13. Ayres H, Ngo H, John AP. Limited changes in activities of daily life performance ability among people with schizophrenia at clinical settings and the factors moderating the changes. Schizophr Res Cogn. 2019;16:29-35.

14. Reichenberg A, Feo C, Prestia D, Bowie CR, Patterson TL, Harvey PD. The course and correlates of everyday functioning in schizophrenia. Schizophr Res Cogn. 2014;1(1):e47-52.

15. Harvey PD, Strassnig M. Predicting the severity of everyday functional disability in people with schizophrenia: Cognitive deficits, functional capacity, symptoms, and health status. World Psychiatry. 2012;11(2):73-9.

16. Strassnig M, Signorile J, Gonzalez C, Harvey PD. Physical performance and disability in schizophrenia. Schizophr Res Cogn. 
2014;1(2):112-21.

17. Aubin G, Chapparo C, Rainville C, Gélinas I, Stip E. Daily activities, cognition and community functioning in persons with schizophrenia. Schizophr Res. 2009;107 (2-3):313-8.

18. Leifker FR, Bowie CR, Harvey PD. Determinants of everyday outcomes in schizophrenia: the influences of cognitive impairment, functional capacity, and symptoms. Schizophr Res. 2009;115(1): 82-7.

19. Bechi M, Bosia M, Spangaro M, Buonocore M, Cavedoni S, Agostoni G, et al. Exploring functioning in schizophrenia: Predictors of functional capacity and real-world behaviour. Psychiatry Res. 2017;251:118-24. 